\title{
Ipsilateral Femoral Neck and Shaft Fracture Management Single Implant versus Multiple Implants
}

\author{
Ahmed AbdElhamid Shamma ${ }^{1}$, Mohammed AbdElaziz Hassan ${ }^{1}$ and Ahmed Mohammed Saber Agour*2 \\ ${ }^{1}$ Department of Orthopedic Surgery, Faculty of Medicine, Al Azhar University, Egypt \\ ${ }^{2}$ Department of Orthopedic Surgery, Elbajour General Hospital,Menoufia, Egypt \\ *Corresponding author: Mohammed Saber Agour, Email:ahmedagour1980, Mobile: (+20)01090497229
}

\begin{abstract}
Introduction: Fracture of shaft and ipsilateral neck of femur is a challenging problem to orthopaedics surgeons. The treatment is often difficult, and there are many protocols for the management of these fractures.

Objectives: To assess the efficiency of multiple implants method for management of ipsilateral fracture neck and shaft femur using plate and screws or retrograde nail for femoral shaft fractures and cannulated screws or sliding hip screw for femoral neck fracture, evaluate the efficiency of reconstruction nail as a single implant method for management of fracture both neck and shaft femur as well as to compare the results of two management methods.

Material and Methods: A total of 20 adult patients with ipsilateral femoral neck and shaft fractures were included in our study. Standard radiographs were obtained. Patients were divided into single implant group (Group I; 10 patients) and multiple implants group (Group II; 10 patients). All patients were followed prospectively for a minimum of 1 year. Fracture union was confirmed radiologically, and functional evaluation was done as per Harris Score.

Results: $70 \%$ of both groups achieved successful fracture union with the remaining $30 \%$ with either nonunion, malunion or necrosis of the femoral head but with non- significant difference between both groups.

Conclusion: Upon comparing single versus multiple implants methods, equivalent results, were clinically and radiologically obtained with both techniques. However, it is difficult to draw a definite conclusion as the number of cases is relatively small. A study with a larger population scale probably gives a definite conclusion.

Keywords: Ipsilateral Femoral Neck, Shaft Fracture, Single implant, Multiple implants.
\end{abstract}

\section{INTRODUCTION}

Fracture of shaft and ipsilateral neck of femur is a challenging problem to orthopedic community. These types of fractures accounts for only $1-9 \%$ of femoral shaft fractures, but these are much more difficult to manage than either injury in isolation and require a modified treatment approach ${ }^{(\mathbf{1})}$.

These injuries usually occur as a result of high energy mechanism such as a motor vehicle crash or fall from height ${ }^{(2)}$. It has been documented that they caused by a longitudinal compression force on a flexed and abducted hip due to the frequency with which the injury is seen in vehicle crash front seat drivers and passengers ${ }^{(3)}$.

Compared to isolated femoral shaft injuries, those fractures with an associated femoral neck injury are more likely to be highly comminuted (Winquist III or IV) indicating a higher energy mechanism. On the other hand, an associated femoral neck fracture is more likely than an isolated neck fracture to be nondisplaced at presentation $(25-60 \%)^{(4)}$. One possible explanation for this is that at the time of injury, the femoral shaft absorbs the majority of the force imparted to the femur resulting in a high degree of shaft comminution but decreasing the amount of force ultimately transferred to the femoral neck ${ }^{(5-6)}$.

Failure to recognize a non-displaced or minimally displaced associated neck fracture prior to fixation of the shaft can lead to displacement, a decrease in neck fixation options, a technically challenging secondary procedure and increased risk of long-term sequelae ${ }^{(\mathbf{1})}$.

Treatment Options to be considered in this type of fractures include; 1-antegrade femoral nailing of the shaft with cancellous screws placed anterior to the nail for fixation of the neck ${ }^{(7)}$. 2-reconstructiontype intramedullary nailing ${ }^{(8)}$.3-various plate combinations [including a sliding hip screw (DHS) or cancellous screws for femoral neck and a plate for the shaft] ${ }^{(9)}$.4-retrograde intramedullary nailing of the shaft and screw fixation of the neck. ${ }^{(10)} 5$ - Long sliding hip screw that can fix both neck and shaft ${ }^{(11)}$.

Each method has its own advantages and disadvantages. The three major issues related to management of these fractures are optimal timing of surgery, which fracture to stabilize first, and the optimal implant to use ${ }^{(\mathbf{1 2})}$.

Most authors recommend prompt, but not emergent, surgery with priority given to anatomic reduction and stabilization of the neck fracture by either closed or open methods. Fixation of the shaft fracture follows as patient condition allows. However some others recommend starting by fixation of the shaft to allow manipulation of the neck by internal and external rotation during reduction ${ }^{(\mathbf{1})}$.

A short delay of 5-6 days in stabilizing ipsilateralund is placed femoral neck and shaft fractures does not seem to affect the ultimate functional outcome ${ }^{(\mathbf{1 3})}$. 


\section{AIM OF THE WORK}

In our study we will do a comparison between single implant versus multiple implants methods for fixation of such a fracture.

\section{MATERIALS AND METHODS}

During the period from February 2016 to May 2018 , this prospective study was conducted enrolling 20 patients with ipsilateral fracture neck and shaft of femur from those visiting Elbajour General Hospital and Elzhar university hospitals.

All cases were arranged into two groups; First group involved 10 cases managed by single implant method by using reconstruction nail and the second group involved another 10 cases managed by double implant method by using plate and screws or retrograde nail for femoral shaft fractures and cannulated screws or sliding hip screw for ipsiateral femoral neck fracture. All patients were followed up prospectively for a minimum period of 12 months.

Our protocol for management consisted of preoperative, intra-operative and post-operative stages. Preoperative stage involved clinical assessment (history, general and local examination), radiological evaluation, preoperative preparation of the patient and patient counseling. Routine hematological investigations were obtained in all patients.

Radiological evaluation was the corner stone for a proper management;it consisted mainly of plain radiographies and $\mathrm{CT}$ scan in four patients.Plain radiography involvedthe standard anteroposterior and lateral views of hip, femur and knee of the affected limb.We used CT scan for the hip joint in selected patients when fracture of femoral neck was suspected in plain X- rays, and in cases when femoral neck fracture was comminuted.

\section{Ethical consideration and Written informed consent:}

An approval of the study was obtained from AlAzhar University academic and ethical committee. Every patient signed an informed written consent for acceptance of the operation.

\section{Surgical procedures:}

All surgical procedures were performed under spinal anesthesia. For the first groupreconstruction nail was introduced for fixation of both neck and shaft femur after placing the patient in lateral position and preparing the appropriate size nail underC-arm guidance ${ }^{(14)}$.

For the second group with non-displaced femoral neck fracture (6 patient), fixation of fracture neck femur was done at first followed by fixation of shaft fracture, while in displaced femoral neck fracture (4 patients); fixation of femoral shaft fracture was done at first followed by fixation of femoral neck fracture. Femoral neck fixation was performed according tothe degree of displacement and anatomical location offemoral neck fracture ${ }^{(15-16-17)}$.

Postoperatively, all patients were followed up on a regular basis. The follow up protocol involved review at the Outpatient clinic at 4, 8 and 12 weeks postoperative. Partial weight bearing was allowed after 8 weeks.Standard radiographs were made for all patients immediately postoperatively, at 6 weeks, 3 months, 6 months and 1 year after surgery. Clinical evaluation was done for hip pain, deformity, range of hip movement as well as return to work. For functional evaluation, Harris hip score was applied ${ }^{(\mathbf{1 8})}$.In the constant method of functional assessment, Harris hip score is based on the assessment of a number of individual subjective and objective parameters in an entirely clinical setting.

\section{RESULTS}

The current study included 20 patients with fracture of shaft and ipsilateral neck of femur. The mean age for group I was $32.2 \pm 7.92$ years while for group II, it was $35.5 \pm 8.58$ years with no statistically significant difference $(\mathrm{P}>0.05)$ as shown in table (1). The majority of cases belonged to male gender and mostly suffered from road traffic accidents (RTA).

Table 1: Socio-demographic criteria of the studied groups

\begin{tabular}{|c|c|c|c|c|}
\hline & \multicolumn{2}{|c|}{ The studied cases } & \multirow{2}{*}{$\begin{array}{l}\mathrm{t}- \\
\text { test }\end{array}$} & \multirow{2}{*}{$\begin{array}{c}P \\
\text { value }\end{array}$} \\
\hline & $\begin{array}{c}\text { Group 1 } \\
\mathbf{N}=\mathbf{1 0}\end{array}$ & $\begin{array}{c}\text { Group } 2 \\
\mathbf{N}=\mathbf{1 0}\end{array}$ & & \\
\hline \begin{tabular}{|l} 
Age \\
$X \pm S D$ \\
Range
\end{tabular} & $\begin{array}{c}32.2 \pm 7.92 \\
20-44\end{array}$ & $\begin{array}{c}35.5 \\
\pm 8.58 \\
23-46\end{array}$ & 0.89 & 0.38 \\
\hline $\begin{array}{l}\text { Sex [n } \\
(\%)] \\
\text { Male } \\
\text { Female }\end{array}$ & $\begin{array}{l}8(80.0) \\
2(20.0)\end{array}$ & $\begin{array}{l}9(90.0) \\
1(10.0)\end{array}$ & $\begin{array}{c}\text { FE } \\
0.39\end{array}$ & 1.0 \\
\hline
\end{tabular}

As regards management procedures, figure 1 demonstrated that the procedure done in group I was reconstruction nail (100\%) but in group II, $60 \%$ was done by cannulated screws + plate, $20 \%$ by cannulated screws + retrograde nail, $10 \%$ by dynamic hip screw + plate and $10 \%$ by proximal femoral locked plate. 
Cannulatedscrews+plate

- Cannulated screws + Retrograde nail
Dynamic Hip Screw + plate

proximal femoral locked plate

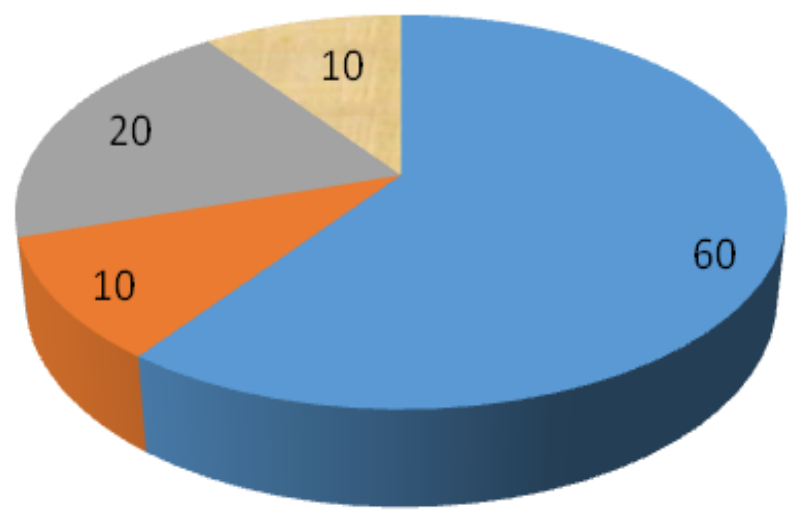

Figure (1): Distribution of the used procedures

Clinical outcomes of the studied cases are illustrated in table 2; all the assessed parameters showed no significant statistical difference between the two studied groups $(\mathrm{P}>0.05)$ except for the duration of rehabilitation $(\mathrm{P}<0.05)$.

Table 2: Comparison of clinical outcome among the studied groups

\begin{tabular}{||l|c|c|c|c||}
\hline \multirow{2}{*}{ Clinical outcome } & \multicolumn{2}{|c|}{ The studied cases } & \multirow{2}{*}{} & \multirow{2}{*}{ P value } \\
\cline { 2 - 5 } & $\begin{array}{c}\text { Group 1 } \\
\mathbf{N = 1 0}\end{array}$ & $\begin{array}{c}\text { Group 2 } \\
\mathbf{N = 1 0}\end{array}$ & FE & 0.63 \\
\hline Hip pain & $2(20.0)$ & $4(40.0)$ & 0.95 & 1.0 \\
\hline Deformity & $1(10.0)$ & $2(20.0)$ & 0.39 & 0.35 \\
\hline Affected range of movement & $2(20.0)$ & $5(50.0)$ & 1.37 & 0.007 \\
\hline $\begin{array}{l}\text { Rehabilitation duration (months) } \\
\text { X } \\
\text { Rang }\end{array}$ & $\begin{array}{c}1.20 \pm 0.42 \\
1-2\end{array}$ & $\begin{array}{c}2.0 \pm 0.67 \\
1-3\end{array}$ & $\begin{array}{c}\mathrm{U} \\
\mathrm{S}\end{array}$ \\
\hline
\end{tabular}

Table 3: Comparison between the two studied groups regarding to radiological outcome

\begin{tabular}{||l|c|c|c|c||}
\hline \multicolumn{1}{||c|}{ Radiological outcomes } & \multicolumn{2}{|c|}{ The studied groups } & Test & $\begin{array}{l}\text { P } \\
\text { value }\end{array}$ \\
\hline & $\begin{array}{l}\text { Group 1 } \\
\mathrm{N}=10\end{array}$ & $\begin{array}{c}\text { Group 2 } \\
\mathrm{N}=10\end{array}$ & & \\
\hline Fracture union without healing problem & $7(70.0)$ & $7(70.0)$ & 0.03 & 1.0 \\
\hline Nonunion & $0(10.0)$ & $2(20.0)$ & 0.0 & 1.0 \\
\hline Malunion & $2(20.0)$ & $1(10.0)$ & 0.75 & 0.46 \\
\hline Avascular necrosis of femoral head & $1(10.0)$ & $0(0.0)$ & 0.05 & 1.0 \\
\hline $\begin{array}{l}\text { Duration of union } \\
\text { X } \pm \text { SD }\end{array}$ & $4.3 \pm 0.95$ & $4.9 \pm 0.99$ & $\mathrm{U}$ & \\
Range & $3-6$ & $3-6$ & & 0.17 \\
\hline
\end{tabular}

$\mathrm{U}=$ Mann Whitney $\mathrm{U}$ 


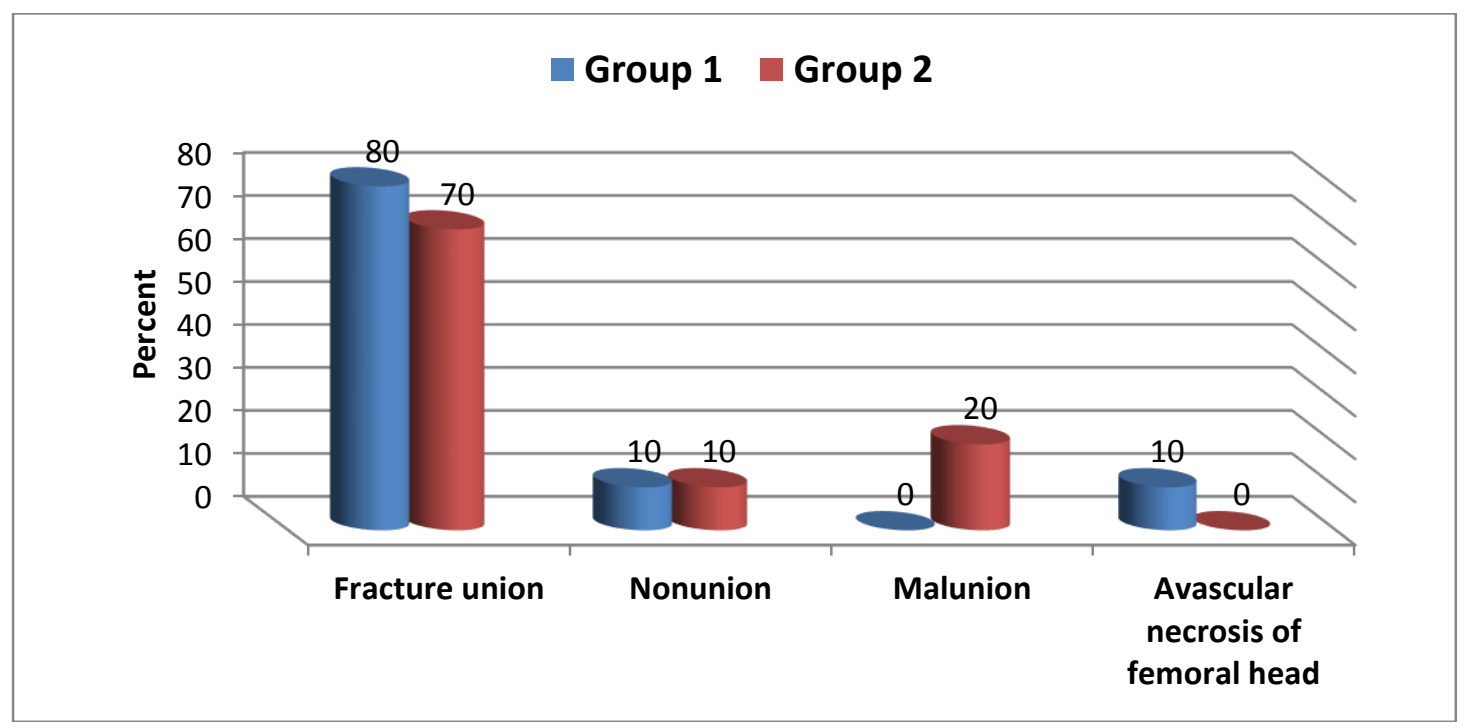

Fig (2) Comparison between the two studied groups regarding to Clinical outcome.

This table 3 and figure (2) demonstrated that $70 \%$ of both groups ended by fracture union with the other $30 \%$ with either nonunion, malunion or necrosis of the femoral head and there is non significant difference between both groups regarding duration of union.

\section{DISCUSSION}

Ipsilateral femoral neck and shaft fractures are challenging. The majority of the patients in the present series were young males with high-energy trauma, as also reported in the literature ${ }^{(2)}$. Femoral neck fractures are most often basilar in our study matching with other series for Jain $\boldsymbol{e t}_{\boldsymbol{a l}} \mathbf{}^{(\mathbf{1 9}) \text {. }}$

Emergency fixation of the fractured neck of femur in this combined injury pattern, unlike isolated femoral neck fractures, may be unnecessary ${ }^{(20)}$. Though there is confusion regarding which fracture should be managed first, there appears to be a general consensus regarding the seriousness of the complications involving femoral neck fractures. In a series utilizing a standard protocol of plate fixations for diaphyseal fractures and lag screws or DHS fixations for the hip fractures, Hung et al.$^{\left({ }^{(12)}\right.}$ reported that the order of fixation of the fractures may not be very important. We stabilized femoral neck fractures first in patients operated with various plate combinations. This protocol is satisfactory in patients with undisplaced neck fractures, as further displacement of the neck fracture is prevented.

There is still no consensus on the optimal treatment method for these complex fractures. In a meta-analysis of the reports published in the literature, the locked intramedullary nails or reconstruction nails yielded results that were superior to those for combinations of plates ${ }^{(20)}$.

The plate series was associated with more frequent infections and nonunion, while the nail fixations were complicated by rotatory malalignments and shortenings ${ }^{(21)}$. However, the difference between the two treatment methods with respect to union, complications and functional outcome was not significant in the present series. The average time for femoral neck and shaft union in the present series was consistent with that reported in other series ${ }^{(22)}$. The choice of the implant in the present series was influenced by the surgeon's preference.

As for union Bose et $\boldsymbol{a l} .{ }^{(\mathbf{2 0})}$ had treated eleven patients with a reconstruction nail, they had multiple associate fracture-healing complications, intraoperative technique issues, and prolonged surgical times of 180 minutes. The authors concluded that the use of this IM nail for this fracture pattern was "demanding" and that technical errors with this implant will lead to fracture complications

In a retrospective analysis of 13 patients with ipsilateral neck and femoral shaft fractures who had healing complications, Watson and Moed ${ }^{(2)}$ reported that $25 \%$ of the femoral neck nonunion that occurred in these 13 patients developed after the use of reconstruction-type intramedullary nails.

Jain et $\boldsymbol{a l}^{\left({ }^{(19)}\right.}$ reported a $20 \%$ incidence of femoral shaft nonunion using reconstruction nailing. Vidyadhara and $\mathbf{R a o}^{(5)}$ reported delayed union of the shaft fracture in12 out of 43 patients. Ahmadet al. ${ }^{(23)}$ documented $10 \%$ nonunion for those having bilateral involvement which required revision nailing with femoral interlocking nail. However, we recorded $20 \%$ nonunion in the same group.

Regarding the femoral neck fractures,Abaloet $\boldsymbol{a l} .{ }^{(24)}$ reported that $92 \%$ of the cases achieved union in a mean duration of 4 (range, 2-5)months, and for femoral shaft fractures, $87 \%$ achieved union in a mean duration of 6 months while Nirmalet al. ${ }^{(25)}$ stated that,all femoral neck fractures united at an average union time of 15 weeks and that two patients had delayed union of femoral shaft fractures. Average union time for fracture shaft of femurs was 20 weeks. Such observations matched with our results as we found 3 cases delayed union 2 of them united after 6 months and one case had done revision after one year 
and we had exchanged retrograde nail by locked broad DCP and bone graft but also didn't unite.

As regards frequency of getting infection, in the first group we had only one stitch infection of the ten patients that had treated medically without debridement,this matched with a series by Nirmalet al. ${ }^{(25)}$.In the second group, Abaloet al. ${ }^{(24)}$ reported high incidence of infection was reported after plating for femoral shaft fractures In his series, 2patients of 40 patients $(5 \%)$ patients developed deep infection, which resolved after debridement and antibiotic treatment. While Khallafet al. ${ }^{(26)}$ in 2005 reported 2-6\% deep infection after fixation of the shaft by plate and screws but in our series we recorded 3 infected cases in the second group two of them was superficial infection treated with dressing and antibiotic and one was deep infection that needed debridement .

As for A.V.NIn the first group we had recorded one case had developed avascular necrosis of the neck of the femur after one year of the surgery and in comparison with literature we found that Nirmalet $\boldsymbol{a l}{ }^{(25)}$ also recorded one case of ten cases complaining of avascular necrosis in his series for the same group of patients while Kao et al. ${ }^{(27)}$, recorded 3-4 \% of his patients had developed avascular necrosis of the head after fixation with reconstruction nail.

However in the second group we didn't get any case complicated with a vascular necrosis matching with Nirmalet al. ${ }^{(25)}$ but against Abaloet al. ${ }^{(24)}$ who recorded 5-7\%as a percentage of AVN postoperative in such a group.

Considering malunion, in the first group we recorded $20 \%$ malunion half of them were in form of coxavaramalnion and one case had mild varus mid shaft femur that were not symptomatic and in literature we found $25 \%$ of varusmalunion recorded by Kao $\boldsymbol{e t}$ $\boldsymbol{a l} .{ }^{(27)}$, however Nirmalet $\boldsymbol{a l} .{ }^{(25)}$ had one case got malunion with coxavara of $98^{\circ}$ and was re-operated later with valgus osteotomy.

In the second group we had one case that had developed coxavara in comparison with other series we found that Abaloet al. ${ }^{(24)}$ had recoded one case developed 6 degreesvarusmalunion from 37 cases in his research while, Nirmalet al. ${ }^{(25)}$ documented $5 \%$ of varusmalunion in his study.

A reconstruction nail is advantageous in terms of possible closed antegrade nailing with minimal incision, and reduced blood loss and biological fixation of both fractures with a single implant this in agreement with Jain et al. ${ }^{(19) .}$. Fixation with plates for the shaft and screws or DHS for the hip is easy from a technical perspective

Biomechanical advantage of reconstruction nailing is outweighed by the technical difficulties involved in accurately placing the proximal screws into the head and neck ${ }^{(\mathbf{1 9}) .}$ In the present series, we also had similar problems in two patients. We are of the opinion that, from a technical point of view, it is much easier to fix with plate and screws or plate and DHS for ipsilateral neck and shaft fracture than with intramedullary nailing with screws or reconstruction nails. Other authors have also had similar opinions as Kao HK, 2004(31).

We performed revision of retrograde nail byexchange with locked plate and bone graft after one year after non union of the shaft that gave good result in union.

The goal of any treatment plan should be anatomic reduction of neck fracture and stable fixation of both fractures, so the patient can be mobilized early (27). Both of the treatment methods used in the present study achieved satisfactory functional outcome in these complex fractures.

The limitations of the present study include the small number of patients in each group and the potential for user bias, because the surgeon could not be blinded with respect to the method used. Reconstruction nailing should not be preferred in displaced femoral neck fractures, because of difficulties in reducing the fracture and its maintenance during nail insertion. We are of the opinion that the femoral neck fracture should preferably be stabilized first. A short delay of 5-6 days in stabilizing ipsilateral femoral neck and shaft fractures does not seem to affect the ultimate functional outcome.

\section{CONCLUSION}

Combined fractures of shaft and neck femur are complex injuries and need judicious evaluation and surgical work up to achieve a good outcome. Fracture fixation with both single and double implants can achieve good union. While each has its own merits and demerits, there is a little consensus which is better. Although in the present study, a good outcome was observed in using both the methods, it is difficult to draw a definite conclusion as the no of cases are comparatively small. A study consisting of more no of cases can give a definite conclusion.

\section{REFERENCES}

1. Boulton CL and Pollak AN (2015): Ipsilateral femoral neck and shaft fractures - Does evidence give us the answer?. Injury Int J Care Injured, 46: 478-483.

2. Watson JT and Moed BR (2002):Ipsilateral femoral neck and shaft fractures: complications and their treatment. ClinOrthopRelat Res., 399:78-86.

3. Tornetta P, HillegassKain MS et al. (2007): Diagnosis of femoral neck fractures in patients with a femoral shaft fracture: improvement with a standard protocol. J Bone Joint Surg., 89:39-43.

4. Randelli P, Landi S, Fanton F et al. (1999): Treatment of ipsilateral femoral neck and shaft fractures with the Russel-Taylor reconstructive nail. Orthopedics, 22(7): 673-6.

5. Vidyadhara S and Rao SK (2009): Cephalomedullary nails in the management of ipsilateral neck and shaft fractures of the femur - one or two femoral neck screws? Injury Journal, 40:296-303. 
6. Alfonso D, Vasquez $O$ and Egol $K$ (2006): Concomitant Ipsilateral Femoral Neck and Femoral Shaft Fracture Nonunion: a Report of Three Cases and a Review of the Literature. Iowa Orthopaedic Journal, 26: 112-18.

7. Datta S, MahendraAS, Christopher LHet al. (2015): Fixation of Shaft and Ipsilateral Neck Fracture of Femur Using Locking Compression Plate and CannulatedCancellous Screws, Journal of Dental and Medical Sciences, 234:27-31.

8. Roop S, Narender KM, Ramchander S et al. (2008):Ipsilateral femoral neck and shaft fractures: a retrospective analysis of two treatment methods, J OrthopaedTraumatol., 9:141-147.

9. Shetty MS, Kumar MA, Ireshanavar SS et al. (2007):Ipsilateral hip and femoralshaftfracturestreatedwith intramedullary nails. IntOrthop., 31:77-81.

10. Wu CC (2004): Ununitedipsilateral femoral neck and shaft fractures: treatment of 16 patients. Arch Orthop Trauma Surg., 124(3):173-178.

11. Oh CW, Oh JK, Park BC et al. (2008):Retrograde nailing with subsequent screw fixation for ipsilateral femoral shaft and neck fractures. J OrthopaedTraumatol., 9:141-147.

12. Hung SH, Hsu CY, Hsu SF et al. (2004): Surgical treatment for ipsilateral fractures of the hip and femoral shaft. Injury, 35:165-169.

13. Weinlein JC (2013): Fractures and dislocations of hip in: Canale ST, Beaty J H. Campbell's operative orthopaedics 4th edition., Mosby Elsevier, Pp. 2725-70.

14. Johnson KD1, Tencer AF, Sherman MC (1987): Biomechanical factore affecting fracture stability and femoral Bursting in closed interamedullary of femoral shaft fracture With illustrative cases presentation. J Orthop Trauma, 1(1):1-11.

15. Crist BD, Khalafi A, Hazelwood SJ et al. (2009):Biomechanical comparison of locked plate with percutaneous insertion cabablity versus angled plate in a subtrochantric fracture Gap model. J Orthop Trauma, 23(9):622-7.

16. Rao JP, Banzon MT, WEISS AB (1983):Rayhack, treatment of unstable intertrochanteric fracture with anatomical reduction and compression hip screw fixation. ClinOrthop., $175: 65-71$

17. Gardner AW, Toh MZ, Lie TT et al. (2015):Cannulatedversus non cannuletedscrew fixation of the femoral neck.Journal of Orthopeadic Surgery, 23(1): 41-6

18. Harris WH (1969): Traumatic arthritis of the hip after dislocation and acetabular fractures: treatment by mold arthroplasty. An end-result study using a new method of result evaluation. J Bone Joint Surg Am., 51(4):737-55.

19. Jain P, Maini L, Mishra Pet al. (2004):Cephalomedullary interlocked nail for ipsilateral hip and femoral shaft fractures. Injury, 35:1031-1038.

20. Bose WJ, Corces A, Anderson LD (1992): A preliminary experience with the Russell-Taylor reconstruction nail for complex femoral fractures. $\mathbf{J}$ Trauma, 32: 71-76.

21. Alho A (1996): Concurrent ipsilateral fractures of the hip and femoral shaft: a meta-analysis of 659 cases. ActaOrthop Scand., 67:19-28.

22. Wu CC (2017): Ununitedipsilateral femoral neck and shaft fractures: treatment of Rajesh RanaIpsilateral fracture neck and shaft of femur: A prospective analysis of two methods. J OrthopTraumatolRehabil., 9:17-20.

23. Ahmad S, ZiaulHSh, Faizan M et al. (2018): Role of reconstruction intramedullary nailing in the treatment of ipsilateral femoral neck and shaft fractures. Saudi J Sports Med., 18:22-6.

24. Abalo A, DossimA, Ouro Bet al. (2008): A Walla Dynamic hip screw and compression plate fixation of ipsilateral femoral neck and shaft Factures Journal of Orthopaedic Surgery, 16(1):35-8.

25. Nirmal CM, Gopal CS, Hung SH et al. (2004): Surgical treatment for ipsilateral fractures of the hip and femoral shaft. Injury, 35:165-169

26. Khallaf F, Al-Mosalamy M, Al-Akkad M et al. (2005): Surgical treatment for ipsilateral fractures of femoral neck and shaft. Med PrincPract., 14:318-24.

Kao HK, Wu CC, Lee PC et al. (2006):Ipsilateral femoral neck and shaft fractures treated with RussellTaylor reconstruction intramedullary nails. Chang Gung Med J., 29(1):79-85. 